\title{
Dielectrons and Direct Virutal Photons at RHIC-STAR
}

\author{
Chi Yang ${ }^{1, \star}$ \\ ${ }^{1}$ Shandong University, Qingdao, China 266237
}

\begin{abstract}
Electromagnetic probes are unique probes of the hot and dense medium created in relativistic heavy-ion collisions due to their minimal interactions with the partonic and hadronic medium. They can be produced at all stages of a collision, thus they provide unique ways to study the medium properties of the whole collision evolution. We present the dielectron invariant mass spectrum at $\sqrt{s_{N N}}=27,39$, and $62.4 \mathrm{GeV}$. Comparing to hadronic cocktail simulation, significant excesses of dielectron invariant mass spectrum at low mass are observed. The direct virtual photon invariant yields derived from the low-mass $e^{+} e^{-}$continuum in $\mathrm{Au}+\mathrm{Au}$ collisions at $\sqrt{s_{N N}}=200 \mathrm{GeV}$ is presented and the excesses from thermal photons in low transverse momentum $\left(p_{T}\right)$ have been observed. Model calculations can simultaneously describe both dielectron low mass excesses and direct virtual photon low $p_{T}$ excesses.
\end{abstract}

\section{Introduction}

Electromagnetic probes such as dielectrons and direct virutal photons are unique probes in the study of the fundamental properties of the hot and dense medium created in ultra-relativistic heavy-ion collisions [1]. Both dielectrons and direct virtual photons are produced in the whole evolution of the system and escape with minimum interaction with the strongly interacting medium. Thus, they provide information about the various stages of the system during the evolution.

In dielectron low mass region (LMR, $M_{e e}<M_{\phi}$ ), the mass spectra of $\rho$ mesons are modified due to their interaction with the medium which is related to the chiral symmetry restoration. At the Relativistic Heavy Ion Collider (RHIC), both STAR and PHENIX Collaborations observed that dilepton mass spectra in $\mathrm{Au}+\mathrm{Au}$ collisions at $\sqrt{s_{N N}}=200 \mathrm{GeV}$ show a significant excess in the LMR when compared to the known hadronic sources [2,3]. Theorectical predictions with many-body approach and a transport model can well describe published STAR measurements of the excess yield in the LMR at $\sqrt{s_{N N}}=200 \mathrm{GeV}[4,5]$. The RHIC Beam Energy Scan (BES) program [6] provides a unique opportunity to systematically test these calculations as a function of the initial collisions energies.

Direct photon stands for all the produced photons except those from hadron decays in the last stage of the collisions. Measurements at the RHIC [7] and the LHC [8] have shown that the production of the high $p_{T}$ direct photons in heavy-ion collisions is consistent with the $p+p$ result scaled by the nuclear overlap function $T_{A A}$ for $p_{T}>5 \mathrm{GeV} / c$, which indicates that the dominant contribution is from hard processes in high $p_{T}$. At $1<p_{T}<3 \mathrm{GeV} / c$, thermal contributions from the hadronic

^e-mail: chiyang@email.sdu.edu.cn 


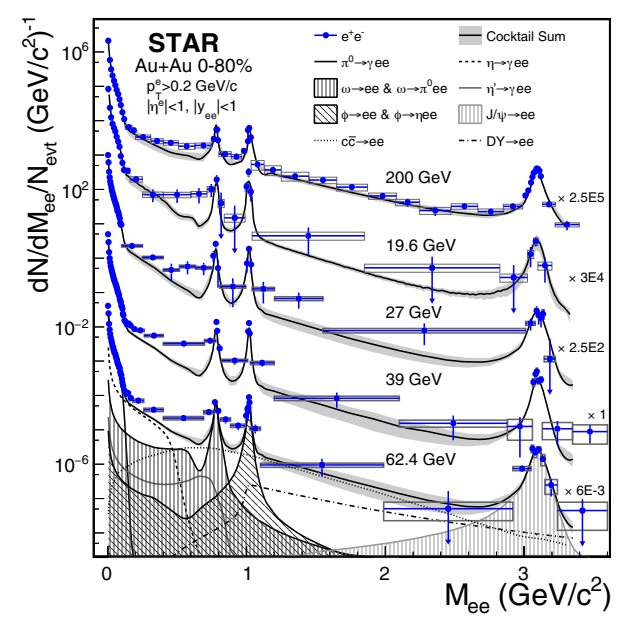

Figure 1. Dielectron invariant mass spectra within the STAR acceptance from $\sqrt{s_{N N}}=19.6,27,39,62.4$, and $200 \mathrm{GeV} \mathrm{0-80 \%} \mathrm{most-central} \mathrm{Au+Au} \mathrm{collisions} \mathrm{[10].}$ The black solid curves represent the hadronic cocktail. The measurements and cocktail predictions are scaled by factors for better presentation.

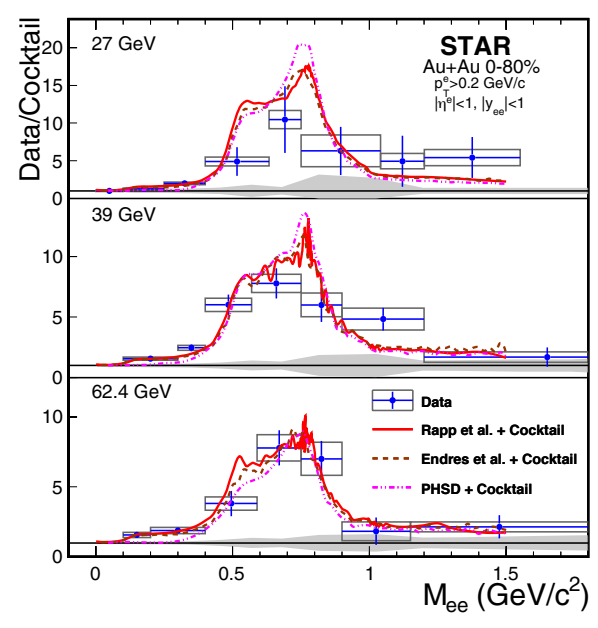

Figure 2. The ratio of the invariant mass spectra to the cocktail with the $\omega$ and $\phi$ yields removed from both the data and cocktail. Model calculations by Rapp et al., Endres et al., and PHSD were separately added to the reference cocktail and compared to the reference cocktail as shown with the curves [10].

medium and Quark-Gluon-Plasma (QGP) play a major role [9]. At $3<p_{T}<5 \mathrm{GeV} / c$, the interaction of high energy partons with the QGP has been predicted to contribute a major part of the direct photon production [9].

In this talk, we will present recent dielectron and direct virtual photon measurements from STAR. The measurements of dielectron production in $\mathrm{Au}+\mathrm{Au}$ collisions with collisions at 27, 39, and 62.4 $\mathrm{GeV}$ are presented [10]. The direct virtual photon invariant yields in the transverse momentum ranges $1<p_{T}<3 \mathrm{GeV} / c$ and $5<p_{T}<10 \mathrm{GeV} / c$ at mid-rapidity derived from the low-mass $e^{+} e^{-}$invariant mass continuum in $0-80 \%$ minimum-bias $\mathrm{Au}+\mathrm{Au}$ collisions at $\sqrt{s_{N N}}=200 \mathrm{GeV}$ are presented [11]. Comparisons to model calculations with thermal contributions from the hadronic medium and QGP are discussed.

\section{Dielectron Results from STAR BES Program}

The dielectron invariant mass spectrum measured within STAR acceptance $\left(p_{T}^{e}>0.2 \mathrm{GeV} / c,\left|\eta^{e}\right|<1\right.$, and $\left|y_{e e}\right|<1$ ) for $0-80 \%$ most-central $\mathrm{Au}+\mathrm{Au}$ collisions at $\sqrt{s_{N N}}=27,39$, and $62.4 \mathrm{GeV}$ are shown in Fig. 1 [10]. The back solid curves represent the hadronic cocktail without the vacuum $\rho$-meson which contribution is expected to be strongly modified in the medium. Results from $0-80 \%$ mostcentral $\mathrm{Au}+\mathrm{Au}$ colllisions at $\sqrt{s_{N N}}=19.6$ and $200 \mathrm{GeV}$ are shown for a systematic study of the initial collisions energy dependence. A significant of yield excess are shown in the LMR relative to hadronic cocktail in all energies. To estimate this yield excess, a ratio of the present data to the hadronic cocktail with yields from $\omega$ and $\phi$ subtracted from both the data and cocktail were shown in Fig. 2. The open boxes and gray bands represent the experimental systematic uncertainties and cocktail simulation uncertainties separately. A clear enhancement is observed in the LMR relative to the hadronic cocktail for each of the three collision energies. Different model calculations within the 


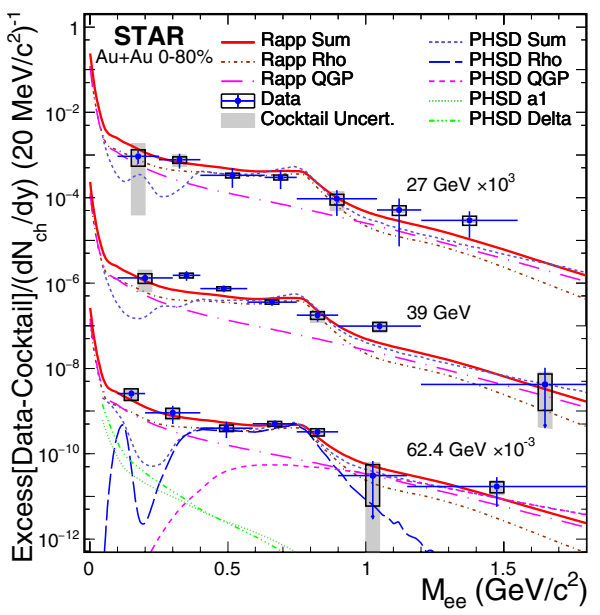

Figure 3. STAR acceptance-corrected dielectron excess invariant mass spectra normalized by $d N_{c h} / d y$ in minimum-bias $\mathrm{Au}+\mathrm{Au}$ collisions at $\sqrt{s_{N N}}=27,39$, and $62.4 \mathrm{GeV}$. Model calculations containing a broadened $\rho$ spectral function in hadronic medium (Rapp Rho) and QGP thermal radiation (Rapp QGP) are also shown.

STAR acceptance are added to hadronic cocktails to compare with measurements. Rapp et al. model is an effective many-body calculation for vector mesons in a QGP where the spectral function of $\rho$ is broadened primarily due to interactions with baryons and meson in a hadron gas. Endres et al. model is a coarse-grained transport approach that includes the $\rho$ spectral function. PHSD is a microscopic transport model which includes the collisional broadening of the $\rho$ meson.

The dielectron excess invariant mass spectra, corrected for detector acceptance, are shown in Figure 3 for minimum-bias $\mathrm{Au}+\mathrm{Au}$ collisions at $\sqrt{s_{N N}}=27,39$, and $62.4 \mathrm{GeV}$. The spectra are normalized by mid-rapidity $d N_{c h} / d y$ to cancel out the volume effect, and compared with model calculations. The model calculations can describe the acceptance-corrected excess invariant mass spectra within uncertainty at different collisions energy range. Model calculations from Rapp et al. in Figure 3 containing a broadened $\rho$ spectral function in hadronic medium (Rapp Rho) and QGP thermal radiation (Rapp QGP) are also shown. The PHSD model calculations in Figure 3 have contributions from the $\rho$ meson (PHSD Rho), QGP (PHSD QGP), Dalitz decays of the a1 (PHSD a1), and $\Delta$ resonances (PHSD Delta). Both models are compared with the excess yield at each energy. Calculations by Rapp et al. have an uncertainty on the order of 15\%, and PHSD model calculations have an uncertainty on the order of $30 \%$. Within uncertainties, the model calculations are found to reproduce the acceptance-corrected excess in $\mathrm{Au}+\mathrm{Au}$ collisions at each of the collision energies.

\section{Direct Virtual Photon Results in Au+Au Collisions at STAR}

Figure 4 shows centrality dependence of the invariant yields of the direct virtual photons in $\mathrm{Au}+\mathrm{Au}$ collisions at $\sqrt{s_{N N}}=200 \mathrm{GeV}$. The $p+p$ result from PHENIX Collaboration is parameterized by a power-law function [12]. The parameterized distribution is then scaled by the nuclear overlap function $T_{A A}$, and compared to the $\mathrm{Au}+\mathrm{Au}$ results in different centralities, as shown in the solid curves. For $1<p_{T}<3 \mathrm{GeV} / c$, the $\mathrm{Au}+\mathrm{Au}$ results are higher than the $T_{A A}$ scaled $p+p$ results, while at $p_{T}>6$ $\mathrm{GeV} / c$ the $\mathrm{Au}+\mathrm{Au}$ yield is consistent with the scaled $p+p$ expectation. However, one should notice 


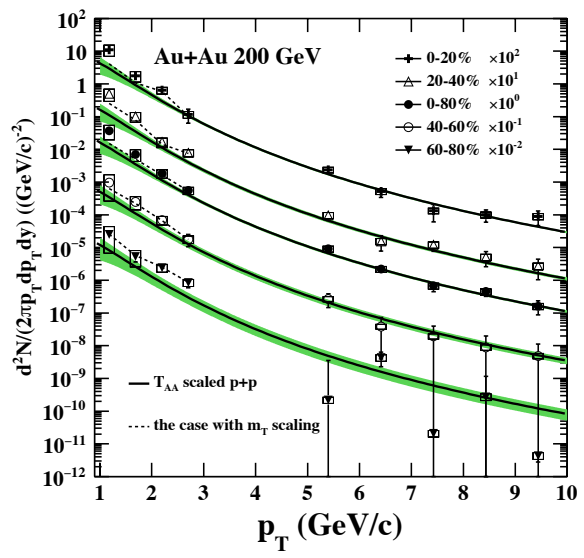

Figure 4. (Color online) Centrality dependence of the direct virtual photon invariant yields as a function of $p_{T}$ in $\mathrm{Au}+\mathrm{Au}$ collisions at $\sqrt{s_{N N}}=200 \mathrm{GeV}$. The solid curves represent a power-law fit to PHENIX $200 \mathrm{GeV}$ $\mathrm{p}+\mathrm{p}$ results, scaled by $T_{A A}$. The bands on the curves represent the uncertainties in the parameterization and in $T_{A A}$. The error bars and boxes represent the statistical and systematic uncertainties, respectively.

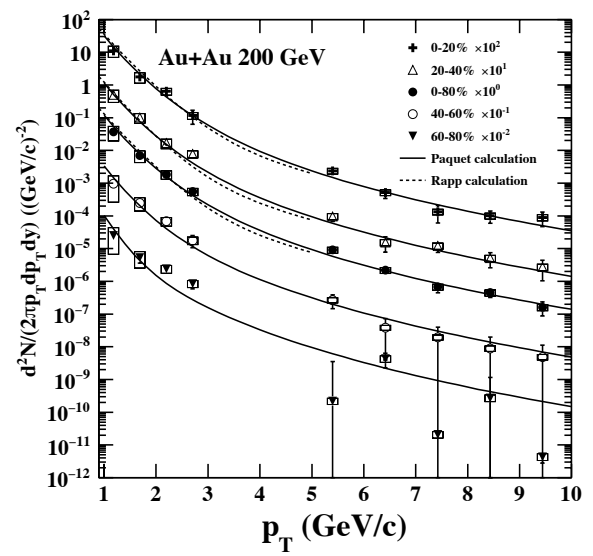

Figure 5. The direct virtual photon invariant yields as a function of $p_{T}$ in $\mathrm{Au}+\mathrm{Au}$ collisions at $\sqrt{s_{N N}}=200 \mathrm{GeV}$ compared to model predictions from Rapp et al. and Paquet et al. . The statistical and systematic uncertainties are shown by the bars and boxes, respectively.

that due to larger uncertainties for data points at $1<p_{T}<2 \mathrm{GeV} / c$ in $40-60 \%$ and $60-80 \% \mathrm{Au}+\mathrm{Au}$ collisions, the measurements at this range are also consistent with the scaled $p+p$ expectations.

A comparison between STAR Au+Au data and model calculations from Rapp et al. and Paquet et al. is shown in Figure 5. Both models include the contributions from QGP thermal radiation, inmedium $\rho$ meson and other mesonic interactions in the hadronic gas, and primordial contributions from the initial hard parton scattering. In Rapp's model, an elliptic thermal fireball evolution is employed for the bulk medium. The sum of the thermal medium and primordial contributions for the former case is shown in Figure 5. In the model from Paquet et al., a (2+1)-D hydrodynamic evolution is employed for the bulk medium. The comparison between the model and data shows that in the $p_{T}$ range $1-3 \mathrm{GeV} / c$ the dominant source is the thermal radiation, while the initial hard-parton scattering becomes dominant in $p_{T}$ range 5-6 GeV/c. The comparison shows agreement between both model calculations and our measurements within uncertainties for all the other centralities except 60-80\% centrality which includes most peripheral collisions, where hydrodynamic calculations might not be applicable.

\section{Summary}

Electromagnetic probes provide unique ways to study the hot and dense medium over the whole evolution. Dielectron invariant mass spectrum in the LMR are observed statistically significant excesses when comparing the data to hadronic cocktails that do not include vacuum $\rho$ decay contributions in $\mathrm{Au}+\mathrm{Au}$ collisions at $\sqrt{s_{N N}}=27,39$, and $62.4 \mathrm{GeV}$. The model calculations with $\rho$ broadening scenario can describes the excess in LMR at all RHIC energies in BES program. The low $p_{T}$ excesses of the direct virtual photon invariant yields compared to $p+p$ references are observed in $\mathrm{Au}+\mathrm{Au}$ 
collisions at STAR. Model calculations can simultaneously describe both dielectron and direct virtual photon results.

\section{Acknowledgement}

The work is partly supported by the Shandong Provincial Natural Science Foundation, China (Grant Number: ZR2019MA005) and the Program of Qilu Young Scholars of Shandong University.

\section{References}

[1] G. David, R. Rapp and Z. Xu, Phys. Rept. 462, 176 (2008).

[2] L. Adamczyk, et al., [STAR Collaboration], Phys. Rev. Lett. 113, 022301 (2014); Phys. Rev. C 92, 024912 (2015).

[3] A. Adare, et al., [PHENIX Collaboration], Phys. Rev. C 93, 014904 (2016).

[4] R. Rapp, Adv. High Energy Phys. 2013, 1 (2013).

[5] O. Linnyk, W. Cassing, J. Manninen, E. L. Bratkovskaya, and C. M. Ko, Phys. Rev. C 85, 024910 (2012).

[6] Experimental Diagram \& Study of Search the QCD for the Phase Critical Point, STAR Note SN0493 (2009), URL: https://drupal.star.bnl.gov/STAR/starnotes/public/sn0493.

[7] S. Afanasiev, et al., [PHENIX Collaboration], Phys. Rev. Lett. 109, 152302 (2012).

[8] J. Adam, et al., [ALICE Collaboration], Phys. Lett. B 754, 235 (2016).

[9] S. Turbide, C. Gale, E. Frodermann, and U. Heinz, Phys. Rev. C 77, 024909 (2008).

[10] J. Adam, et al., [STAR Collaboration], arXiv:1810.10159.

[11] L. Adamczyk, et al., [STAR Collaboration], Phys. Lett. B 770, 451 (2017).

[12] S.S. Adler, et al., [PHENIX Collaboration], Phys. Rev. Lett. 98, 012002 (2007) . 